\title{
Comparación de los métodos de escalas y frecuencia de comportamiento para valorar la competencia de innovación. El punto de vista de alumnos y profesor en el caso de una asignatura de máster
}

Juan A. Marin-Garcia ${ }^{a}$, Lucia Ramirez Bayarri ${ }^{b}$ y $^{\text {a }}$ Angeles Andreu Andres ${ }^{\text {c }}$

${ }^{a}$ ROGLE. Dpto. de Organización de Empresas. Universitat Politècnica de València. Camino de Vera S/N 46021 Valencia. jamarin@omp.upv.es, 'Department d'Ensenyament de la Generalitat de Catalunya. luraba@gmail.com, ${ }^{c}$ Departamento de Idiomas. Universitat Politècnica de València. maandreu@idm.upv.es

\begin{abstract}
Skills assessment is a complex task that includes deciding on various aspects or dimensions of competencies. Moreover, it is not always easy for teachers to observe and assess certain skills. It is therefore recommended sometimes incorporate students as evaluators of their own abilities. Self-assessment has benefits because it increases the critical observation and student capacity. Similarly, it encourages reflection and meta-cognition, which are two important components in independent learning throughout life. However, despite the benefits cited, students supposed them a challenge, assigning a rating using a score on a scale of 0-10, not all students are willing to participate as evaluators, nor are they equally reliable. This research compares two different scales for the collection of data for self-assessment of competence of innovation and teamwork with 16 master students. We base or comparison on the degree of agreement between the scores on both scales. We also use the qualitative opinions of the students.
\end{abstract}

Keywords: self-assessment of students; higher education; self-evaluation; innovation competency

\begin{abstract}
Resumen
La evaluación de competencias es una tarea compleja que incluye decidir sobre varios aspectos o dimensiones de la competencia. Por otra parte, no siempre es fácil que los profesores puedan o sepan observar y valorar determinadas habilidades. Por ello, se ha recomendado, en ocasiones, incorporar a los alumnos como evaluadores de sus propias habilidades. La auto-evaluación presenta beneficios pues aumenta la capacidad crítica y de observación de los estudiantes. Del mismo modo, fomenta la reflexión y la meta-cognición, que son dos componentes importantes en el aprendizaje autónomo a lo largo de la vida. No obstante, a pesar de los beneficios citados,
\end{abstract}


Comparación de los métodos de escalas y frecuencia de comportamiento para valorar la competencia de innovación. El punto de vista de alumnos y profesor en el caso de una asignatura de máster

a los estudiantes les supone un reto, el asignar una nota usando una puntuación en una escala de 0 a 10 y no todos los alumnos están dispuestos a participar como evaluadores, ni son igual de fiables cuando evalúan. Esta investigación compara dos escalas diferentes para la recogida de datos para la auto-evaluación de la competencia de innovación y trabajo en equipo de los 16 estudiantes de una asignatura de master basándose en el grado de acuerdo de las puntuaciones obtenidas por ambas escalas y la valoración crítica de los estudiantes.

Palabras clave: Evaluación de estudiantes; educación universitaria; autoevaluación; competencia de innovación; comparación de métodos

\section{Introducción}

Una de las competencias más demandadas en las sociedades avanzadas es la competencia de innovación, que aparece reflejada en la mayoría de los planes de estudios de España bajo diferentes nombres: innovación, creatividad, emprendimiento, habilidad para tomar decisiones, capacidad para resolver problemas...(Andreu Andrés and García-Casas, 2014, Ingols and Shapiro, 2014, Marin-Garcia et al., 2008). En este trabajo nos hemos basado en el modelo de competencia de innovación representado en la Figura 1, que es una evolución de trabajos previos (Marin-Garcia et al., 2013, Marin-Garcia et al., 2010, Marin-Garcia et al., 2011).

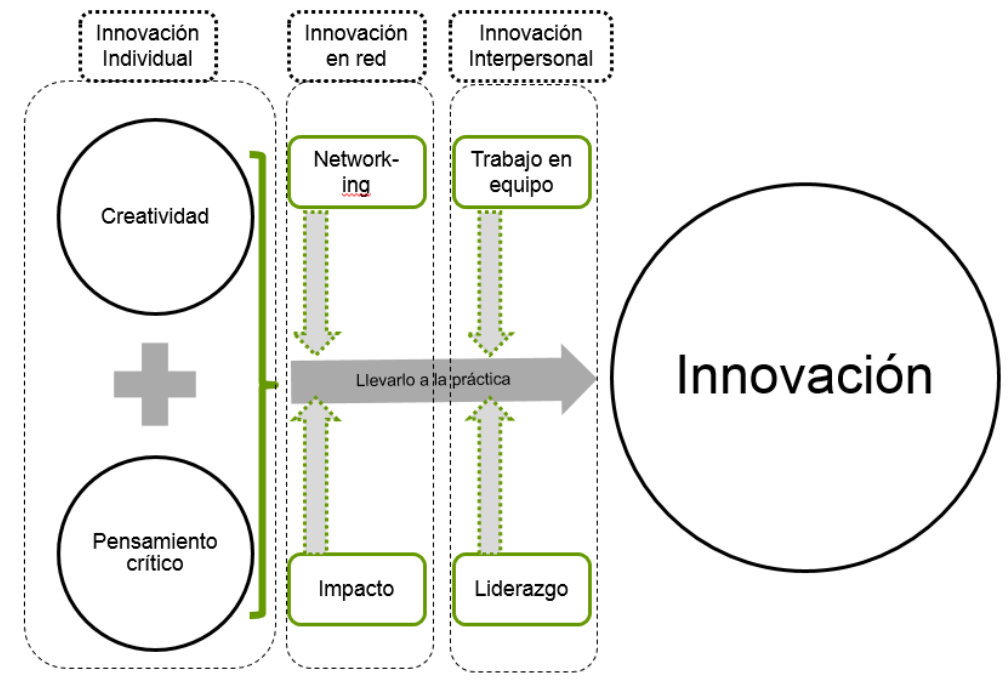

Figura 1.- Modelo de innovación (Fuente: Marin-Garcia (2015) sin publicar) 
La evaluación de competencias es una tarea compleja que incluye decidir sobre varios aspectos o dimensiones de la competencia. En general, para la mayoría de las competencias profesionales, no suele haber demasiado acuerdo acerca de qué subdimensiones (capacidades o habilidades) las componen y, por lo tanto, qué habilidades deben evaluarse (Lohmann and Prumper, 2006, Marin-Garcia et al., 2013).

Por otra parte, no siempre es fácil que los profesores puedan o sepan observar y valorar determinadas habilidades a través de los comportamientos de sus estudiantes. La evaluación del comportamiento se centra en "identificar aspectos claramente observables y el modo en que la persona interactúa con su entorno" (Groth-Marnat, 2003). Los principales instrumentos para la evaluación del comportamiento son los cuestionarios y estrategias de observación (Dowdy et al., 2013). En esta comunicación, nos vamos a centrar en los primeros, en su modalidad de cuestionario autoadministrado por el propio estudiante que se autoevaúa (Groth-Marnat, 2003). Tanto la auto-evaluación como la evaluación de compañeros presenta beneficios pues aumenta la capacidad crítica y de observación de los estudiantes (Van Overveld and Verhoeff, 2013). Del mismo modo, fomenta la reflexión y la meta-cognición, que son dos componentes importantes en el aprendizaje autónomo a lo largo de la vida (Lind et al., 2002, Ljungman and Silén, 2008). No obstante, a pesar de los beneficios citados, a los estudiantes les supone un reto, el asignar una nota y no todos los alumnos están dispuestos a participar como evaluadores, ni son igual de fiables cuando evalúan (Pond, 2007, Van Overveld and Verhoeff, 2013).

Dentro de las diferentes tradiciones en las que se ha analizado la evaluación por observación (psicología clínica, pedagogía, gestión de recursos humanos), parece haber un consenso en que hay otro aspecto importante en el proceso de evaluación, además de quién hace la medición. Es el modo en que se mide (las escalas o instrumentos utilizados). No es nuestro objetivo en este trabajo hacer un resumen de las ventajas y limitaciones de la observaciones de comportamiento frente a las escalas de calificación, la frecuencia con las que se usan o las recomendaciones para la puesta en práctica en general de esta estrategia de evaluación. Mucha de esa información se pueden encontrar en Dowdy et al (2013) o en Marin-Garcia et al. (2012). Nosotros nos centraremos en describir los diferentes tipos de escalas que podemos usar para evaluar competencias por medio de cuestionarios autoadministrados y compararemos los resultados de dos de ellas.

Podemos considerar cuatro posibles métodos de recogida de los datos de entrada necesarios para la evaluación de los alumnos. Las listas ordenadas (rankings), las escalas de puntuación (ratings), las escalas basadas en observación de frecuencia de comportamientos (BARS y BOS) y la comparación pareada (paired comparisons) (Dowdy et al., 2013, Hatzinger and Dittrich, 2012, Marin-Garcia et al., 2012). En este trabajo nos vamos a centrar en el segundo y tercero de ellos.

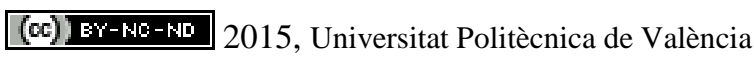

Congreso In-Red (2015) 
Comparación de los métodos de escalas y frecuencia de comportamiento para valorar la competencia de innovación. El punto de vista de alumnos y profesor en el caso de una asignatura de máster

Las escalas de puntuación se caracterizan por que las categorías de respuesta, compartidas por diferentes preguntas o ítems en el cuestionario, se identifican con números o distancia respecto a límites o anclas verbales (Figura 2).

numerical rating scale
QUICK REFERENCE
Any rating scale in which the response categories are identified by numbers, such as a scale from -3 to +3 or from 0 to 10 . The main
alternatives are rating scales with unlabelled response categories, indicated by a row of boxes, dashes, or other symbols; verbal
rating scales, and graphic rating scales.
From: numerical rating scale

Verbal rating scale
QUICK REFERENCE
Any rating scale in which the response categories are labelled with words, such as Strongly Disagree, Disagree, Neutral, Agree,
Strongly Agree. Compare graphic rating scale, numerical rating scale.
From: verbal rating scale in A Dictionary of Psychology»

\section{graphic rating scale}

QUICK REFERENCE

Any rating scale in which a response is indicated by marking a position on a line, such as one anchored by strongly disagree at one end and strongly agree at the other, or some other analogue (2) representation of the dimension being rated. Compare numerical rating scale, verbal rating scale.

From: graphic rating scale $\mathrm{O}$ in A Dictionary of Psychology»

Figura 2: Escalas de evaluación

(fuente:http://www.oxfordreference.com/view/10.1093/oi/authority.20110803095904227?rskey= $\underline{\text { VE2Fjz\&result }=3 \text { ) }}$

Por otra parte, las escalas de evaluación ancladas en comportamientos (BARS), identifican los aspectos claves de un puesto de trabajo o posición (por ejemplo, estudiante) y las posibles conductas que pueden mostrar al desarrollar sus tareas, ordenándolas desde las más ineficientes o indeseables hasta las más eficientes o deseables (Figura 3) 


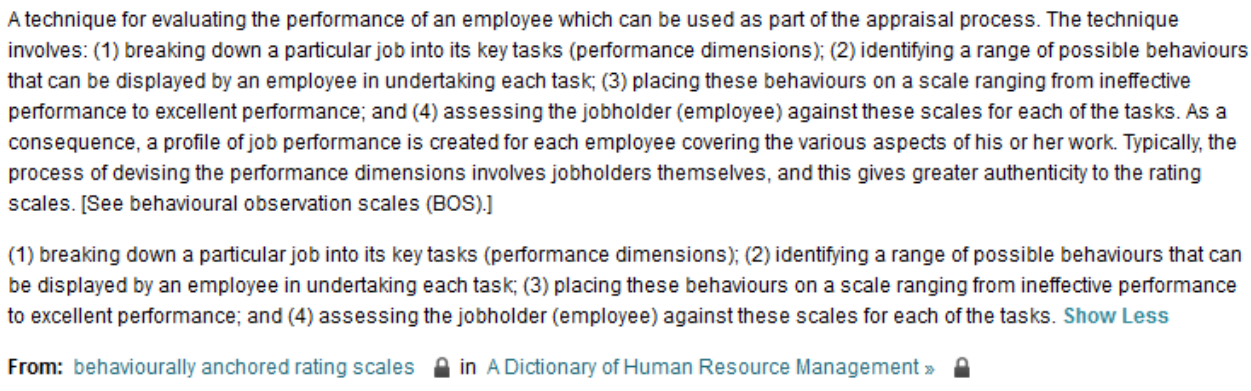

(1) breaking down a particular job into its key tasks (performance dimensions); (2) identifying a range of possible behaviours that can be displayed by an employee in undertaking each task; (3) placing these behaviours on a scale ranging from ineffective performance to excellent performance; and (4) assessing the jobholder (employee) against these scales for each of the tasks. Show Less

From: behaviourally anchored rating scales $\mathrm{a}$ in A Dictionary of Human Resource Management »

Figura 3.- Behaviourally anchored rating scales (BARS) (Fuente: http://www.oxfordreference.com/view/10.1093/oi/authority.20110803095456792?rskey=cWbwX 8\&result $=3$ )

Por último, las escalas de observación de comportamiento (BOS) identifican una serie de conductas observables en los alumnos, como en BARS, pero se recogen datos de la frecuencia en que los estudiantes muestran esas conductas para conseguir un adecuado rendimiento en las tareas encargadas durante el curso. Son especialmente adecuadas cuando se quiere evaluar el proceso no solo el resultado o la productividad de los estudiantes (Figura 4)

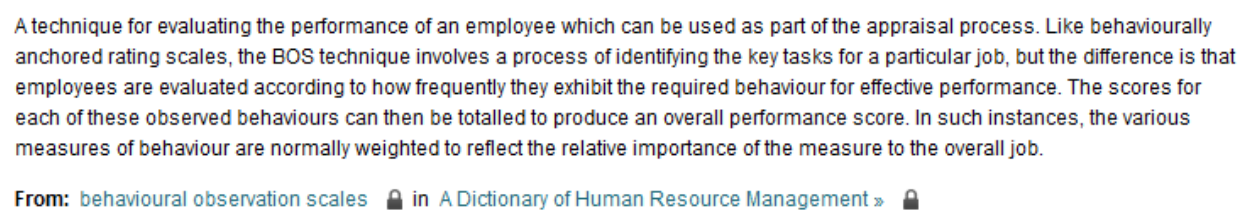

Figura 4.- Behavioural observation scale (BOS) (Fuente: http://www.oxfordreference.com/view/10.1093/oi/authority.20110803095456852)

La ponderación, en las diferentes dimensiones, de los valores obtenidos en las escalas de puntuación o de las frecuencias de las alternativas de comportamientos observados permite obtener una calificación global, que puede usarse para ordenar a los estudiantes de mejor a peor rendimiento.

\section{Objetivos}

El objetivo de esta investigación es comparar dos escalas diferentes para la recogida de datos para la auto-evaluación de la competencia de innovación y trabajo en equipo de los estudiantes de una asignatura de master basándose, por un lado, en el grado de acuerdo de las puntuaciones obtenidas por ambas escalas y por otro la valoración crítica de los estudiantes acerca de cuál de las dos opciones les ha resultado más sencillo de contestar si creen que los

\section{(cc) EY-NC-ND 2015, Universitat Politècnica de València}

Congreso In-Red (2015) 
Comparación de los métodos de escalas y frecuencia de comportamiento para valorar la competencia de innovación. El punto de vista de alumnos y profesor en el caso de una asignatura de máster

resultados reflejan bien cómo son respecto a la innovación y al trabajo en equipo y si les ha ha resultado útil rellenar el cuestionario

\section{Desarrollo de la innovación}

La asignatura donde se ha realizado la intervención es de un máster internacional oficial, su principal objetivo de aprendizaje está relacionado con las competencias de innovación y de trabajo en equipo, tiene 16 alumnos matriculados. La asignatura se impartió en inglés, siendo la mitad de los alumnos de habla castellana y la otra mitad proveniente de intercambio de diferentes países europeos (con especial incidencia de Alemania). Los datos fueron recogidos a principios de septiembre de 2014, por medio de una plataforma de encuestas web (limesurvey) siendo las preguntas mostradas en castellano e inglés. La propia plataforma web grababa también el tiempo invertido por los estudiantes para completar su auto evaluación.

La segunda semana de clase se solicitó a los alumnos que se autoevaluaran usando el cuestionario con 25 preguntas con formato escala de evaluación de 11 niveles de respuesta (de 0 a 10), pero con 6 anclas verbales (desde “no observado” as “excelente”). Se decidió usar anclas verbales y 11 niveles de respuesta basándonos en trabajos previos que avalan que las propiedades psicométricas de las escalas mejoran cuando se utilizan anclas verbales y cuando hay más de 7-9 niveles en la comparación (De Beuckelaer et al., 2013, Yiu et al., 2007), ya que proporcionar suficiente rango de variabilidad, sin complicar en exceso el proceso de comparación (ver Figura 5). Los 25 ítems se promedian para calcular las puntuaciones en 3 dimensiones de la innovación (individual -12 ítems-, interpersonal -8 ítems- y en red -5 ítems-).

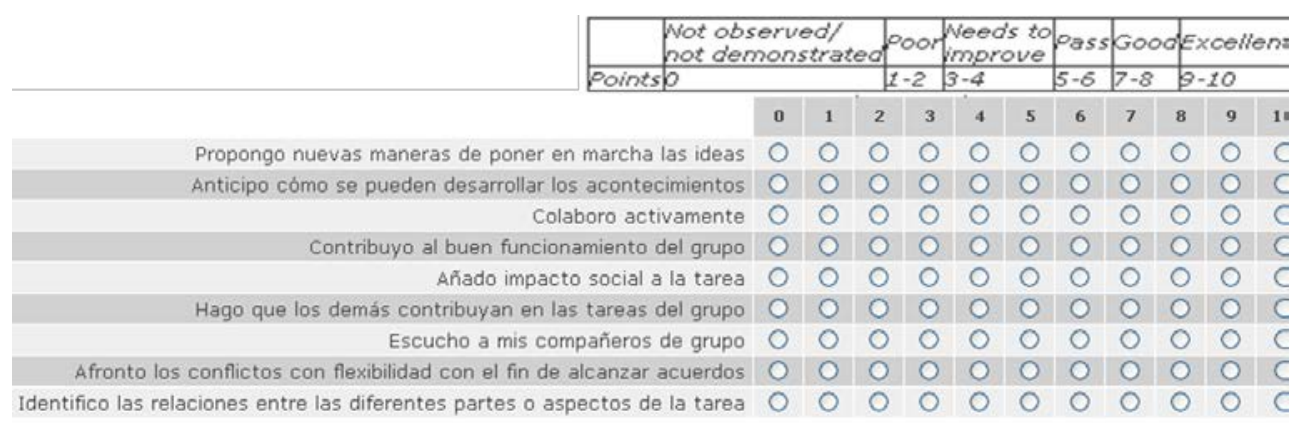

Figura 5.- Extracto de las escala de valoración usada en la primera toma de datos (Watts et al., 2013, Watts et al., 2012)

Una semana más tarde (tiempo que se consideró suficiente para evitar efecto recuerdo, pero al mismo tiempo no excesivo para garantizar que los alumnos no habían tenido tiempo para modificar su competencia de innovación), se les solicitó a los mismos alumnos que se volvieran a evaluar, pero usando una escala BOS. Este autodiagnóstico se componía de 18 grupos de preguntas derivadas de las 25 originales, cada una de ellas con 5 comportamientos 
ordenados desde menos favorecedor de la conducta innovadora a más favorecedor. Las respuestas eran la frecuencia en la que se mostraba cada uno de los comportamientos. Las puntuaciones de los 5 comportamientos de cada pregunta se ponderaban para calcular la puntuación de la pregunta y, posteriormente, las 18 preguntas se agrupaban para calcular las puntuaciones de las 3 dimensiones de innovación (del mismo modo que se hace en la versión "escala de puntuación"). Se ha pasado a 18 grupos de preguntas, pues en las 25 preguntas originales había ítems que representaban comportamientos de diferentes niveles de una misma pregunta en BOS. En la versión BOS, están presentes las 25 preguntas originales como anclas de un determinado nivel de comportamiento favorecedor de la innovación. Es decir, ambos cuestionarios pretenden medir lo mismo, pero de diferente manera. En la Figura 6 se presentan los grupos de comportamientos asociados a dos de las preguntas "creatividad" e "impacto".
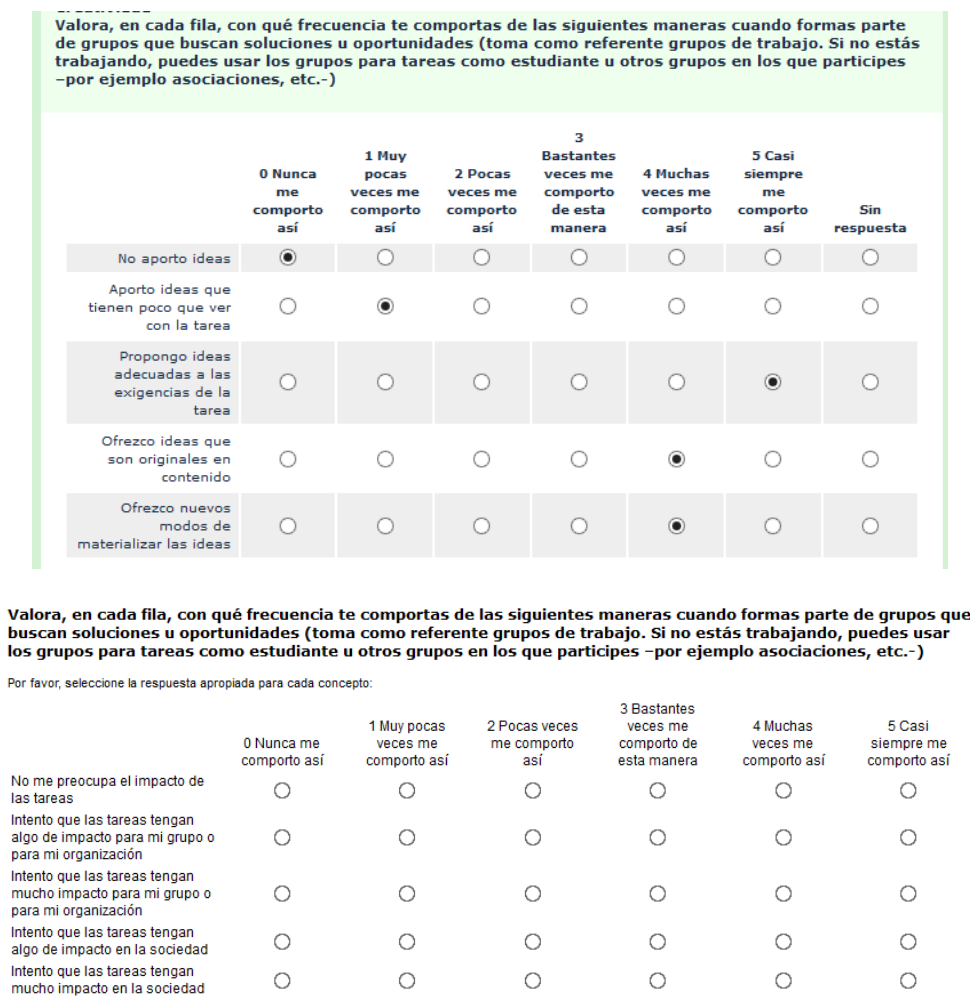

Figura 6.- Extracto de la escala usada en la segunda toma de datos 
Comparación de los métodos de escalas y frecuencia de comportamiento para valorar la competencia de innovación. El punto de vista de alumnos y profesor en el caso de una asignatura de máster

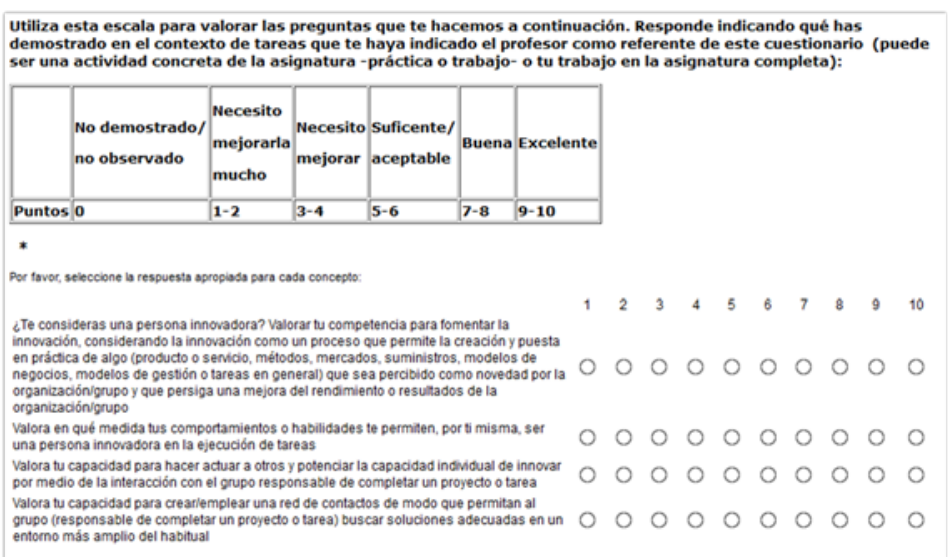

Figura 7.- Escalas monoitem generales de la innovación

El grado de acuerdo de las puntuaciones de los alumnos entre las dos versiones se computará con los coeficientes de correlación y la prueba de muestras T pareadas (Hair et al., 1999).

El análisis cualitativo de las percepciones de los estudiantes está basado en las respuestas a estas preguntas abiertas:

- Cuál de las dos opciones, "escala de puntuación” (normal) o BOS, te ha resultado más sencillo de contestar y por qué.

- Una vez completado cada cuestionario te llega un informe con tu puntuación:

- ¿Eran parecidos los resultados en las dos versiones?

o ¿Crees que los resultados reflejan bien cómo eres respecto a la

innovación y al trabajo en equipo? ¿En las dos opciones, sólo en una (cuál), ninguna? ¿Por qué?

- ¿ ¿Te ha resultado útil rellenar el cuestionario? ¿Cuál más? ¿Por qué?

El análisis se ha realizado siguiendo un protocolo de codificación sistemática soportado por el programa informático ATLAS-TI (Bringer et al., 2006, Friese, 2012).

\section{Resultados}

El tiempo invertido en completar el autodiagnóstico está en torno a 5 minutos en la versión escalas de puntuación y de 18 minutos en la versión BOS (Tabla 1). Esto es debido a que el número de valoraciones que se hacen en la versión BOS es más elevado (18 preguntas, cada una de ellas con 5 comportamientos a valorar su frecuencia).

Tabla 1.- Tiempo invertido en cada una de las versiones (minutos)

(c)) EY-NC-ND 2015, Universitat Politècnica de València 
Juan A. Marin-Garcia, Lucia Ramirez Bayarri y $M^{a}$ Angeles Andreu Andres

\begin{tabular}{|c|c|c|c|c|c|}
\hline & $N$ & Minimum & мaximum & Mean & Std. Deviation \\
\hline InterviewTime & 16 & 2,08 & 9,84 & 4,39 & 2,23 \\
\hline interviewtime_BOS & 16 & 9,28 & 29,78 & 18,38 & 6,00 \\
\hline
\end{tabular}

A continuación hemos analizado las medias y correlaciones entre las puntuaciones obtenidas en las dos tomas de dato. En principio, nuestra hipótesis era que ambas medidas debían ser iguales pues en una semana el alumno no ha cambiado sustancialmente los comportamientos asociados a la competencia evaluada. Los cuatro primeros pares de comparación se hacen con exactamente las cuatro mismas preguntas a modo de test-retest (Figura 7). Al alumno se le pide que se evalúe con una escala de 0 a 10 (no demostrado a excelente) su competencia de innovación en general (SingleItemInnov y SingleItemInnov_BOS), su capacidad para la innovación individual (SingleItemIndiv y SingleItemIndiv_BOS), su capacidad para la innovación en grupo (SingleItemInter y SingleItemInter_BOS) , y su capacidad para la innovación a través de una red de contactos (SingleItemNetw y SingleItemNetw_BOS). Los resultados (Tabla 2, Tabla 3 y Tabla 4) muestran que no hay diferencias significativas en las medias entre los dos momentos y que las correlaciones entre las medidas son altas (sobre todo en la capacidad de innovación interpersonal y en res). Por ello nos servirá como evidencia de que el alumno se percibe sin apenas cambios en cuento innovación.

Veamos qué ocurre con las puntuaciones en las dos versiones de los cuestionarios (Pair 5 a Pair 7 en las tablas). En la dimensión individual, no hay diferencias significativas entre las puntuaciones de los dos tipos de cuestionarios, aunque la correlación es moderada. En las otras dos dimensiones, a pesar de que existen diferencias significativas, la correlación entre las medidas es elevada. En la versión BOS los alumnos se puntúan con menos puntos, pero la posición de las personas, de mayor a menor puntuación, se mantiene. Es decir, parece que la versión escala de puntuación, se satura más fácilmente que la versión BOS.

Tabla 2.-Descriptivos

\begin{tabular}{|c|c|c|c|}
\hline & & Mean & Std. Deviation \\
\hline \multirow[t]{2}{*}{ Pair 1} & SingleItemInnov & 6,81 & 1,471 \\
\hline & SingleItemInnov_BOS & 6,19 & 1,834 \\
\hline \multirow[t]{2}{*}{ Pair 2} & SingleItemIndiv & 7,13 & 1,360 \\
\hline & SingleItemIndiv_BOS & 6,69 & 1,778 \\
\hline \multirow[t]{2}{*}{ Pair 3} & SingleItemInter & 7,06 & 1,914 \\
\hline & SingleItemInter_BOS & 7,13 & 1,784 \\
\hline \multirow[t]{2}{*}{ Pair 4} & SingleItemNetw & 7,00 & 2,066 \\
\hline & SingleItemNetw_BOS & 6,81 & 1,905 \\
\hline \multirow[t]{2}{*}{ Pair 5} & ICBIndividual & 72,08 & 9,01 \\
\hline & ICBIndividual_BOS100 & 73,51 & 10,75 \\
\hline \multirow[t]{2}{*}{ Pair 6} & ICBInterpersonal & 78,43 & 8,84 \\
\hline & ICBInterpersonal_BOS100 & 71,31 & 11,68 \\
\hline \multirow[t]{2}{*}{ Pair 7} & ICBRed & 73,87 & 17,89 \\
\hline & ICBRed_BOS100 & 59,22 & 14,31 \\
\hline
\end{tabular}

(cc)) EY-NC-ND 2015, Universitat Politècnica de València

Congreso In-Red (2015) 
Comparación de los métodos de escalas y frecuencia de comportamiento para valorar la competencia de innovación. El punto de vista de alumnos y profesor en el caso de una asignatura de máster

Tabla 3.-correlaciones Pearson

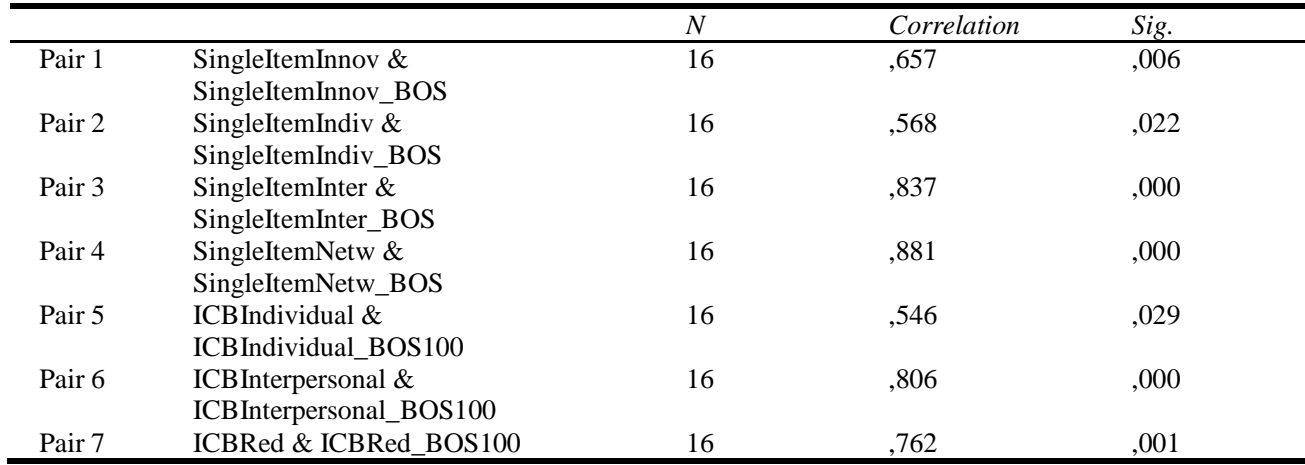

Tabla 4.- Paired Samples Test (95\% intervalo de confianza) 15 df; Sig 2 tailed

\begin{tabular}{|c|c|c|c|c|c|c|c|}
\hline & & $\begin{array}{l}\text { Mean } \\
\text { diference }\end{array}$ & $\begin{array}{l}\text { Std. } \\
\text { Deviation }\end{array}$ & Lower & Upper & $t$ & Sig. \\
\hline $\begin{array}{l}\text { Pair } \\
1\end{array}$ & $\begin{array}{l}\text { SingleItemInnov - } \\
\text { SingleItemInnov_BOS }\end{array}$ & 625 & 1,408 &,- 125 & 1,375 & 1,775 & ,096 \\
\hline $\begin{array}{l}\text { Pair } \\
2\end{array}$ & $\begin{array}{l}\text { SingleItemIndiv - } \\
\text { SingleItemIndiv_BOS }\end{array}$ & 438 & 1,504 &,- 364 & 1,239 & 1,163 & 263 \\
\hline $\begin{array}{l}\text { Pair } \\
3\end{array}$ & $\begin{array}{l}\text { SingleItemInter - } \\
\text { SingleItemInter_BOS }\end{array}$ &,- 063 & 1,063 &,- 629 & ,504 &,- 235 & 817 \\
\hline $\begin{array}{l}\text { Pair } \\
4\end{array}$ & $\begin{array}{l}\text { SingleItemNetw - } \\
\text { SingleItemNetw_BOS }\end{array}$ & 188 & 981 &,- 335 & ,710 & ,764 & 456 \\
\hline $\begin{array}{l}\text { Pair } \\
5\end{array}$ & $\begin{array}{l}\text { ICBIndividual - } \\
\text { ICBIndividual_BOS100 }\end{array}$ & $-1,432$ & 9,538 & 6,515 & 3,650 &,- 601 & ,557 \\
\hline $\begin{array}{l}\text { Pair } \\
6\end{array}$ & $\begin{array}{l}\text { ICBInterpersonal - } \\
\text { ICBInterpersonal_BOS100 }\end{array}$ & 7,123 & 6,943 & 3,423 & 10,823 & 4,104 & ,001 \\
\hline $\begin{array}{l}\text { Pair } \\
7\end{array}$ & $\begin{array}{l}\text { ICBRed - } \\
\text { ICBRed_BOS100 }\end{array}$ & 14,654 & 11,618 & 8,463 & 20,845 & 5,045 & ,000 \\
\hline
\end{tabular}

En las contestaciones abiertas, se manifiesta un acuerdo de los estudiantes en que ambas versiones ofrecen unos resultados similares (Figura 8). Esos resultados son percibidos como muy válidos, porque la escala de 0 a 100 recibida coincide con su percepción del despliegue de las diferentes dimensiones de su competencia de innovación (Figura 9). También hay unanimidad en considerar el autodiagnóstico como una actividad muy útil en la asignatura, porque les ayuda a la reflexión de sus comportamientos; motiva a aprender y detectar áreas fuertes o áreas de mejora (Figura 10). Aparece en algunos casos una interesante solicitud de que no se limite a auto-diagnóstico sino que les parecería muy útil contar con la visión de terceras personas (Figura 11). Por último, todos coinciden en considerar que la versión “escala” es mucho más breve que la versión BOS pero eso no siempre significa que sea más fácil de contestarla. Para algunos alumnos, la versión BOS es más fácil de contestar, aunque sea más larga, porque es más descriptiva y les permite ser más precisos y claros en sus contestaciones. Además, algunas personas consideran más detallada o rica la información que le ofrece la versión BOS (Figura 11).

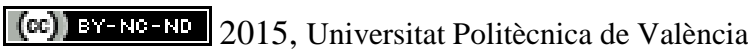


Juan A. Marin-Garcia, Lucia Ramirez Bayarri y $M^{a}$ Angeles Andreu Andres

\begin{tabular}{l} 
[1:35] En las dos versiones he \\
obtenido resulta.. \\
\hline En las dos versiones he obtenido \\
resultados parecidos,
\end{tabular}

$[1: 31]$ ambos resultados eran bastante
parejos,
$\begin{aligned} & \text { ambos resultados eran bastante } \\ & \text { parejos, }\end{aligned}$

[1:17] Resulta que en la encuesta normal mi pun..

Resulta que en la encuesta normal mi puntuación es parecida a la encuesta bar.

Figura 8.- algunos ejemplos representativos de las contestaciones de los alumnos sobre comparación
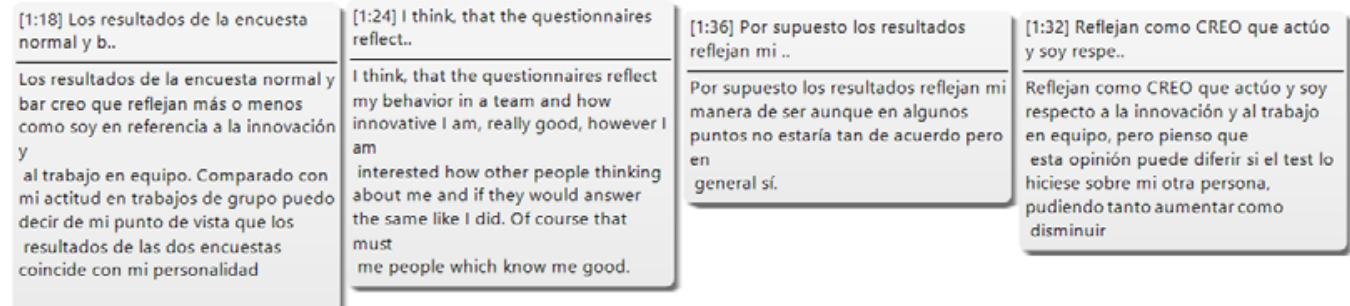

Figura 9.- algunos ejemplos representativos de las contestaciones de los alumnos sobre acierto del diagnóstico

\begin{tabular}{l} 
[1:20] La encuesta me ha animado \\
pensar sobre m.. \\
\hline $\begin{array}{l}\text { La encuesta me ha animado pensar } \\
\text { sobre mis actividades en grupos. Así } \\
\text { podía reflejar mis } \\
\text { competencias y me he dado cuenta } \\
\text { de unos puntos positivos y debiles. }\end{array}$ \\
\hline [1:27] me ha resultado útil rellenar los \\
cuesti.. \\
\hline me ha resultado útil rellenar los \\
cuestionarios, porque de esa forma \\
puedo ver cual es mi \\
comportamiento a la hora de trabajar \\
en equipo y, a la misma vez, \\
motivarme y mejorar en los aspectos \\
que flojee \\
un poco más, para finalmente \\
conseguir tener un buen \\
comportamiento de interacción, \\
iniciativa, innovación y \\
compañerismo
\end{tabular}

\begin{tabular}{|c|c|}
\hline $\begin{array}{l}{[1: 26] \text { In my opinion it was meaningful }} \\
\text { to fill .. }\end{array}$ & $\begin{array}{l}{[1: 19] \text { Me parece muy útil e }} \\
\text { interesante para mi.. }\end{array}$ \\
\hline $\begin{array}{l}\text { In my opinion it was meaningful to fill } \\
\text { out the questionnaires, because it } \\
\text { gives you the possibility to think about } \\
\text { yourself and how behave in an } \\
\text { environment. However I think it would } \\
\text { be good, if other people fill the same }\end{array}$ & $\begin{array}{l}\text { Me parece muy útil e interesante para } \\
\text { mi misma rellenar la encuesta. Todos } \\
\text { hemos trabajado varias veces en } \\
\text { grupos. Pero yo he trabajado en } \\
\text { grupos sin darme realmente cuenta de } \\
\text { mi actitud. }\end{array}$ \\
\hline $\begin{array}{l}\text { would be interesting if my self- } \\
\text { perception is the same like other }\end{array}$ & $\begin{array}{l}\text { [1:33] Siempre es útil rellenar } \\
\text { cuestionarios d.. }\end{array}$ \\
\hline about me. & $\begin{array}{l}\text { Siempre es útil rellenar cuestionarios de } \\
\text { este tipo para ver que visión se tiene de } \\
\text { uno mismo, aunque insisto en }\end{array}$ \\
\hline [1:14] Por eso creo que los cuestiona.. & $\begin{array}{l}\text { que vería más útil aún esta tarea si otras } \\
\text { personas con las que he formado } \\
\text { equipo lo rellenasen por mi, para }\end{array}$ \\
\hline $\begin{array}{l}\text { Por eso creo que los cuestionarios son } \\
\text { muy útil para evaluar su mismo para } \\
\text { ver que hay una área donde puedo } \\
\text { mejorarme, } \\
\text { pero no para mandos que quieren }\end{array}$ & $\begin{array}{l}\text { de ver las cosas y así poder } \\
\text { mejorar. Aun que ello conlleva mucho } \\
\text { más trabajo }\end{array}$ \\
\hline
\end{tabular}

saber que persona tiene que mejorar aciones de los alumnos sobre utilidad

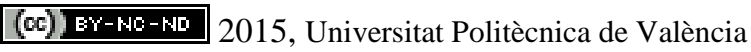

Congreso In-Red (2015) 
Comparación de los métodos de escalas y frecuencia de comportamiento para valorar la competencia de innovación. El punto de vista de alumnos y profesor en el caso de una asignatura de máster

\begin{tabular}{|c|c|c|}
\hline $\begin{array}{l}\text { [1:34] La más sencilla me ha resultado } \\
\text { Normal y.. }\end{array}$ & $\begin{array}{l}\text { [1:21] Lo que pasa es que a veces la } \\
\text { gente se c.. }\end{array}$ & \multirow[b]{2}{*}{$\begin{array}{l}\text { [1:23] The questionnaire "BARS" of course is lo.. } \\
\text { The questionnaire "BARS" of course is longer and consist of } \\
\text { more questions. The first "single-item" is the same like } \\
\text { in the normal questionnaire. Up the diagnostic part it goes } \\
\text { more into detail, how I behave by different tasks, also in } \\
\text { special environments. For me this is easier to answer, } \\
\text { because I really can image a situation and answer more } \\
\text { correctly }\end{array}$} \\
\hline $\begin{array}{l}\text { La más sencilla me ha resultado } \\
\text { Normal ya que son preguntas mas } \\
\text { faciles de contestar y más cortas. Solo } \\
\text { tienes que } \\
\text { interiorizar y pensar como eres y } \\
\text { responder abiertamente }\end{array}$ & $\begin{array}{l}\text { Lo que pasa es que a veces la gente se } \\
\text { cansa cuando tiene que conestar } \\
\text { muchas preguntas. A } \\
\text { continuación puede haber una } \\
\text { influencia a la contestación a las } \\
\text { preguntas. Sin embargo creo que con } \\
\text { la encuesta } \\
\text { bar se puede sacar más información } \\
\text { de una persona }\end{array}$ & \\
\hline \multirow{3}{*}{$\begin{array}{l}\text { he questionnaire "normal" refers how I } \\
\text { see myself without seeing aspects of } \\
\text { the environment. In the diagnostic } \\
\text { part you really have to estimate your } \\
\text { behavior. That was kind of difficult for } \\
\text { me, because sometimes my self - } \\
\text { perception is different like other } \\
\text { people would describe me. And if I am } \\
\text { honest I belong to this group of } \\
\text { people, who } \\
\text { often underestimate their own skills. } \\
\text { The questionnaire was completely just } \\
\text { about me and how I behave. }\end{array}$} & $\begin{array}{l}\text { [1:16] En general la encuesta normal } \\
\text { me parece.. }\end{array}$ & \multirow[b]{2}{*}{ [1:30] Puede que la opción normal, al ser más c.. } \\
\hline & En general la encuesta normal me & \\
\hline & $\begin{array}{l}\text { parece bastante sencillo de contestar. } \\
\text { De un lado se puede decir que es muy } \\
\text { corta y tarda poco en hacer. De otro } \\
\text { lado las preguntas están } \\
\text { comprensibles. Además la encuesta } \\
\text { está bien } \\
\text { estructurada y el orden de las } \\
\text { preguntas me parece útil. Aunque } \\
\text { está corto tiene un valor informativo. } \\
\text { Este aspecto }\end{array}$ & $\begin{array}{l}\text { Puede que la opción normal, al ser más corta y más concisa } \\
\text { sea más sencilla de contestar. A pesar de esto la } \\
\text { opción BARS me parece mejor ya que te permite un rango } \\
\text { más "amoldable" de opciones y por tanto, a mi } \\
\text { juicio es más precisa. En cualquier caso, ambos son } \\
\text { complejos de contestar ya que las preguntas formuladas, } \\
\text { en bastantes casos, deberían ser resueltas por terceras } \\
\text { personas respecto a uno }\end{array}$ \\
\hline
\end{tabular}

Figura 11.- algunos ejemplos representativos de las contestaciones de los alumnos sobre comparación

\section{Conclusiones}

Hemos presentado un modelo para evaluar la competencia transversal de innivación (según el modelo UPV también aparecerían representadas las competencias de creatividad, liderazgo, pensamiento crítico y trabajo en equipo) con estudiantes universitarios en asignaturas de máster con pocos alumnos. El modelo ha demostrado ser válido, con una carga de trabajo razonable y asumible por alumnos, sin excesivas complejidades a la hora de implantarlo y proporcionando unos resultados fiables y muy útiles para los alumnos.

Consideramos que las principales contribuciones de nuestro trabajo son:

- Comparar la fiabilidad de las puntuaciones basadas en ratings y en BOS, así como su viabilidad en un contexto poco trabajado (la docencia universitaria).

- Para los docentes universitarios, analizamos las ventajas e inconvenientes de la aplicación de ambos método de toma de datos, para resolver el problema de la evaluación de competencias o habilidades de los alumnos que tienen que ver con el proceso de grupo y no con los resultados o productos del grupo.

\section{Limitaciones e investigación futura:}

La principal limitación es que hemos analizado un grupo de 16 alumnos de máster, lo que nos impide generalizar estos resultados a grupos numerosos o de alumnos de grado. También hemos considerado el mismo peso para los diferentes ítems que componen las escalas o dimensiones de las competencias observadas, de modo que las dimensiones con más criterios, pesan más en la nota ponderada. 
Para investigación futura sería interesante ampliar la muestra a más alumnos de master y grado, de diferentes titulaciones y comprobar si, por un lado las opiniones unánimes en la muestra usada en esta investigación, se siguen manteniendo en otras muestras. Además habría que estimar la proporción de alumnos que prefieren cada tipo de autodiagnóstico (pues aparecen diferentes opiniones en nuestra investigación). También debería comprobarse que la supuesta utilidad de opiniones de terceras personas que conocen a los alumnos se confirma cuando realmente sean evaluados por conocidos.

Otra línea de trabajo que consideremos interesante sería hacer un análisis entre los ítems equivalentes en ambos cuestionarios. Los 25 ítems de la versión escala de puntuación están incluidos en la versión BOS pero los niveles de respuesta son frecuencia de manifestación de comportamiento en lugar de grado de excelencia en el comportamiento. Podría comprobarse el efecto de la forma de respuesta en la media y distribución de las respuestas.

\section{Agradecimientos}

Este trabajo ha sido realizado con la financiación de la Unión Europea ["FINCODA" proyecto 554493-EPP-1-2014-1-FI-EPPKA2-KA] (The European Commission support for the production of this publication does not constitute an endorsement of the contents which reflects the views only of the authors, and the Commission cannot be held responsible for any use which may be made of the information contained therein) y PIME/2014/A/013/ABarómetro INCODE: Evaluación de Competencias de Innovación en la Empresa y en la Universidad.

\section{Referencias}

ANDREU ANDRÉS, M. A. \& GARCÍA-CASAS, M. (2014) La evaluación de la participación en equipos de trabajo universitarios (Assessment of participation in higher education team working activities). WPOM-Working Papers on Operations Management, 5.

BRINGER, J. D., JOHNSTON, L. H. \& BRACKENRIDGE, C. H. (2006) Using Computer-Assisted Qualitative Data Analysis Software to Develop a Grounded Theory Project. Field Methods, 18, pp. 245266.

DE BEUCKELAER, A., TOONEN, S. \& DAVIDOV, E. (2013) On the optimal number of scale points in graded paired comparisons. Quality and Quantity, 47, pp. 2869-2882.

DOWDY, E., TWYFORD, J. \& SHARKEY, J. D. (2013) Methods of Assessing Behavior: Observations and Rating Scales. IN D. H. SAKLOFSKE, V. L. SCHWEAN \& C. R. REYNOLDS (Eds.) The Oxford Handbook of Child Psychological Assessment. (Oxford University Press).

FRIESE, S. (2012) Qualitative Data Analysis with ATLAS.ti, (London, SAGE Publications Ltd).

GROTH-MARNAT, G. (2003) Handbook of psichological assessment, (Hoboken, New Jersey, John Wiley \& Sons, Inc.).

HAIR, J. F., ANDERSON, R. E., TATHAM, R. L. \& BLACK, W. C. (1999) Análisis de datos multivariante [Multivariate data analysis], (Madrid, Prentice Hall).

HATZINGER, R. \& DITTRICH, R. (2012) Prefmod: An R package for modeling preferences based on paired comparisons, rankings, or ratings. Journal of Statistical Software, 48.

INGOLS, C. \& SHAPIRO, M. (2014) Concrete Steps for Assessing the Soft Skills in an MBA Program. Journal of Management Education, 38, pp. 412-435. 
Comparación de los métodos de escalas y frecuencia de comportamiento para valorar la competencia de innovación. El punto de vista de alumnos y profesor en el caso de una asignatura de máster

LIND, D. S., REKKAS, S., BUI, V., LAM, T., BEIERLE, E. \& COPELAND, I. I. I. (2002) Competency-Based Student Self-Assessment on a Surgery Rotation. Journal of Surgical Research, 105, pp. 31-34.

LJUNGMAN, A. G. \& SILÉN, C. (2008) Examination involving students as peer examiners. Assessment \& Evaluation in Higher Education, 33, pp. 289-300.

LOHMANN, A. \& PRUMPER, J. (2006) Questionnaire for direct participation in the office (FdP-B) results concerning its reliability and validity. Zeitschrift fur Arbeits-und Organisationspsychologie, 50, pp. 119-134.

MARIN-GARCIA, J. A., AZNAR-MAS, L. E. \& GONZÁLEZ-LADRÓN-DE-GEVARA, F. (2011) Innovation types and talent managment for innovation. Working Papers on Operations Management, 2, pp. 25-31.

MARIN-GARCIA, J. A., BAUTISTA POVEDA, Y., GARCIA-SABATER, J. J. \& VIDAL CARREAS, P. I. (2010) Implantación de la innovación continua en la gestión de operaciones: una revisión de la literatura. Innovar, 20, pp. 77-95.

MARIN-GARCIA, J. A., GARCIA-SABATER, J. J., MAHEUT, J., VALERO-HERRERO, M. \& ANDRES-ROMANO, C. (2012) Gestión de recursos humanos para ingenieros de la rama industrial, (Harlow, Pearson Education).

MARIN-GARCIA, J. A., GARCIA-SABATER, J. P., MIRALlES, C. \& RODRÍGUEZ VILLALOBOS, A. (2008) Profile and competences of Spanish industrial engineers in the European Higher Education Area (EHEA). Journal of Industrial Engineering and Management, 1, pp. 269-284. MARIN-GARCIA, J. A., PEREZ-PEÑALVER, M. J. \& WATTS, F. (2013) How to assess innovation competence in services: The case of university students. Direccion y Organizacion, pp. 48-62.

POND, K. (2007) Student Experiences of Peer Review Marking of Team Projects. International Journal of Management Education, 6, pp. 30-43.

VAN OVERVELD, K. \& VERHOEFF, T. (2013) Self-consistent peer ranking for assessing student work: Dealing with large populations. CSEDU 2013 - Proceedings of the 5th International Conference on Computer Supported Education.

WATTS, F., GARCIA-CARBONELL, A. \& ANDREU ANDRÉS, M. A. (2013) Innovation competencies development: INCODE barometer and use guide, (Turku, Turku University od Applied Sciences).

WATTS, F., MARIN-GARCIA, J. A., GARCIA-CARBONELL, A. \& AZNAR-MAS, L. E. (2012) Validation of a rubric to assess innovation competence. Working Papers on Operations Management, 3, pp. 61-70.

YIU, E., CHAN, K. \& MOK, R. (2007) Reliability and confidence in using a paired comparison paradigm in perceptual voice quality evaluation. Clinical Linguistics and Phonetics, 21, pp. 129-145. 